\title{
LE ROMAN QUÉBÉCOIS ET SES ADAPTATIONS : ÉCART DE L'ORIGINAL ET DISSIMULATION IDÉOLOGIQUE
}

\author{
Katarzyna WóJCIK \\ Université de Varsovie
}

\begin{abstract}
En): Film adaptation of the novel illustrate different relations of the film to the source text: the complete fidelity, transformations consequent on the passage from literature to film, modifications of the text's signification on the esthetic and ideological level. Quebec literature and cinema are sensible to the non-artistic discourses present in the Quebec society. For these reason film adaptation of Quebec novel can be considered as an example of "ideological dissimulation" according to the political and social context contemporary to the realization of the movie. The adaptation can either extenuate the novel's ideological signification or use a canonical text to deliver its own message reinforced by the film relation to the literary original. The analyze of two novels, considered as classical, of Quebec literature - Bonheur d'occasion (1945) by Gabrielle Roy and Maria Chapdelaine (1916) by Louis Hémon - and their filmic adaptations by Claude Fournier (Bonheur d'occasion, 1983) and Gilles Carle (Maria Chapdelaine, 1983) illustrates these different strategies of "ideological dissimulation".
\end{abstract}

Keywords (En): adaptation ; film ; novel ; ideology ; Quebec

Mots-clés (Fr) : adaptation ; cinéma ; roman ; idéologie ; Québec

\section{Littérature, cinéma, idéologie}

La littérature et le cinéma au Québec sont particulièrement sensibles à la situation politique, sociale et historique qui les entoure. Petr KYLOUŠEK met en relief cette «perméabilité » de la littérature du Québec au non-littéraire : «[...] plus que les autres littératures, la littérature canadienne-française et québécoise est "impure", "contaminée" dès ses origines, car le discours esthétique, littéraire s'y mélange et sert de support à d'autres discours » (2009: 25). De même, Christian POIRIER insiste sur la présence des discours non artistiques, en particulier identitaires, au cinéma québécois et l'inscription des films dans l'idéologie de l'époque de leur réalisation. Selon le chercheur, «[...] il semble exister, au Québec, un lien étroit entre cinéma et politique, entre le cinéma et l'identité collective québécoise d'une part, et le contexte politique global, d'autre part » (2004 : 2). Si le cinéma québécois est, lui aussi, marqué par la conjoncture sociale et politique ainsi que par la problématique identitaire, cela vient de sa situation « minoritaire ». Comme le remarque Gilles DELEUZE (1985 : 283-284), dans le cas des collectivités minoritaires en crise d'identité, le cinéma non seulement s'adresse à une communauté mais aussi la fonde et la problématique identitaire ainsi que les questions politiques ressortent au premier plan ${ }^{1}$.

\footnotetext{
${ }^{1}$ Gilles Deleuze se réfère ici à la notion de « littérature mineure » qu'il a définie, avec Félix Guattari, en reprenant l'idée de Kafka (DeleuZe, GuatTARi, 1984).
} 
L'idéologie constitue un de ces discours qui « contaminent» la littérature et le cinéma. Sans oublier la spécificité du contexte québécois, province francophone sur le continent anglophone, on peut inscrire la réflexion sur l'inscription du discours idéologique dans l'œuvre littéraire et filmique dans le contexte plus général des études littéraires inspirées par la sociocritique. En précisant les intérêts de la sociocritique, Claude DUCHET remarque que « [1]'œuvre n'est lue, ne prend figure, n'est écrite qu'au travers d'habitudes mentales, de traditions culturelles, de pratiques différenciées de la langue, qui sont les conditions de la lecture » (1971 : 8). Attentive au «co-texte », c'est-à-dire à «tout ce qui se dit et s'écrit dans un état de société » (ANGENOT, 1989:83), la sociocritique s'intéresse à l'inscription de l'idéologie dans le texte littéraire et insiste sur la nécessité de prendre en compte les facteurs extérieurs au texte, tout en soulignant leur caractère discursif ${ }^{2}$. Selon Régine RoBIN (1989: 7-32), l'idéologie peut être présente dans le texte littéraire comme le «projet idéologique » de l'auteur qui veut exprimer ses idées dans un texte concret, comme le «cadre idéologique de départ », c'est-à-dire le système des valeurs dominant dans la société et par rapport auquel se positionne l'écrivain, comme l' « idéologie de référence » que l'auteur adopte dans la vie sans l'exprimer nécessairement dans un texte donné et enfin, comme l' «idéologie du texte », celui-ci pouvant fonctionner indépendamment des intentions de l'auteur.

Ce lien que la littérature et le cinéma entretiennent avec d'autres discours devient d'autant plus intéressant si l'on considère le passage de la littérature au cinéma sous forme d'adaptation. La relation du texte artistique à l'idéologie se complique : l'adaptation se réfère non seulement aux discours inscrits dans le texte littéraire qu'elle met sur l'écran mais aussi à ceux qui lui sont contemporains. Elle est ainsi doublement significative : d'une part comme une œuvre autonome qui se positionne face au discours social ${ }^{3}$ et au contexte socio-historique, politique et culturel de sa réalisation; d'autre part en tant que prise de parole sur un texte antérieur.

La modification de la portée idéologique du roman lors de sa mise sur l'écran devient d'autant plus visible si le texte adapté est bien connu du public ${ }^{4}$. Ainsi, les adaptations des œuvres devenues des «classiques» de la littérature québécoise semblent des exemples particulièrement intéressants pour notre analyse. Les deux romans choisis - Bonheur d'occasion (1945) de Gabrielle Roy et Maria Chapdelaine (1916) de Louis Hémon - peuvent être considérés comme de tels textes «canoniques ». Euvres marquantes pour l'histoire littéraire et le discours identitaire du Québec, ces romans ont été adaptés en 1983, respectivement par Claude Fournier et par Gilles Carle. Bonheur d'occasion de Fournier et Maria Chapdelaine de Carle participent à la vague d'adaptations cinématographiques du

\footnotetext{
${ }^{2}$ Voir la distinction entre le «contexte » et le «co-texte» et la définition du «co-texte»par Marc ANGENOT (1989: 83).

${ }^{3}$ Micheline CAMBRon considère le « discours social » ou « récit fondamental commun » (1989: 43) comme un discours d'une société à un moment donné, par rapport auquel les auteurs prennent position, soit pour le soutenir, soit pour s'y opposer.

${ }^{4}$ Alicja Helman (1998: 15) remarque que même si les spectateurs sont conscients du caractère inévitable des transformations lors de la mise du roman sur l'écran, leur connaissance de l'original littéraire devient un cadre de référence incontournable pendant le visionnage du film.
} 
cinéma québécois des années 1980 et s'inscrivent ainsi dans des stratégies commerciales de l'industrie cinématographique ${ }^{5}$. En même temps, ces films se positionnent de manière différente par rapport aux romans qu'ils mettent sur l'écran.

\section{Adaptation cinématographique : lecture et recontextualisation}

Les adaptations cinématographiques des romans québécois entretiennent différentes relations avec leurs textes sources. Elles peuvent aller de la « fidélité » ${ }^{6}$ presque totale au texte littéraire jusqu'aux films dont le lien avec l'original romanesque relève plutôt de l'inspiration ou de l'allusion. Il serait d'ailleurs difficile de parler de la reprise complète du roman par le film, vu que les transformations sont indissociables du processus d'adaptation (GAUDREAULT, MARION, 2008 : 14). Les modifications viennent de la nécessité de «s'adapter» au contexte nouveau, qu'il soit socioculturel, historique ou «médiatique ». André GAUDREAUlT et Philippe MARION montrent les différents aspects de ce changement de contexte pouvant «[...] représenter un autre public, une autre communauté culturelle de réception (au sens géographique, socioculturel ou historique), mais [...] aussi désigner un changement de genre et surtout un changement de média [...]» $(2008: 15)^{7}$.

En fait, à part des transformations imposées par la transposition du discours littéraire en récit filmique ${ }^{8}$, on note les modifications de sens au niveau esthétique et idéologique apportées par les réalisateurs qui s'adressent à un autre public dans une autre époque et une autre situation socioculturelle. Selon Michel SERCEAU, «l'adaptation n'est pas seulement une transposition [...] mais un mode de réception et d'interprétation des thèmes et formes littéraires »(1999: 9-10). L'adaptation constitue la recontextualisation de l'œuvre littéraire qui est lue selon de nouveaux codes culturels et dans un contexte différent. Dans ce sens, elle peut être considérée comme révélatrice de l'imaginaire collectif de l'époque de sa réalisation :

\footnotetext{
${ }^{5}$ Yves LEVER (1988 : 290) remarque qu'une des tendances dans la cinématographie québécoise après 1981 est la réalisation des «superproductions » pour le cinéma accompagnées d'un format pour la télévision. Les titres cités, parmi lesquels Bonheur d'occasion est évoqué à côté de Les Plouffe, Maria Chapdelaine, Crime d'Ovide Plouffe, Le Matou, sont tous des adaptations des romans québécois.

${ }^{6}$ André Gaudreault et Philippe Marion considèrent la notion de fidélité comme un «point aveugle de toutes les conceptions de l'adaptation » (2008: 13) et une «idée-réflexe » (2008:13) qu'il faut abandonner lors de l'étude des adaptations.

7 André Gaudreault et Philippe MARION insistent sur le caractère «intermédiatique » de l'adaptation et proposent de considérer le changement de média comme un contexte nouveau : « un autre média est, à cet égard, une 'situation nouvelle', à laquelle il faut accoutumer le texte que l'on souhaite adapter » (2008: 15). Pourtant, l'analyse de l'inscription du discours idéologique dans les films inspirés des romans prendra en compte surtout les autres contextes évoqués.

${ }^{8}$ L'adaptation est souvent définie, selon la terminologie de Roman Jakobson, comme traduction intersémiotique qui exige le transfert du système des signes linguistiques vers le système des signes audiovisuels. Voir HOPFINGER (1974 : 79).
} 
L'adaptation témoigne en tous cas du dialogue qu'entretiennent une époque, une catégorie socioculturelle, une société, avec la substance vive de la littérature. [...] Lecture de l'œuvre littéraire, aspect de sa fortune et de son influence, elle en est une autre forme de sémiotisation, où s'inscrit et se structure une part non négligeable de l'imaginaire de l'homme d'aujourd'hui.

(SERCEAU, $1999: 10)$

La reprise du texte littéraire dans un autre contexte permet de considérer l'adaptation comme un texte nouveau dont la signification s'établit pourtant par rapport au texte de départ. GAUDREAULT et MARION proposent la notion d' « intentionnalité adaptative » qu'ils définissent comme « une volonté marquée [...] de modifier l'hypotexte» (2008: 19). L'adaptation est ainsi vue comme « discours intentionnel » $(2008: 20)$ et son étude devrait prendre en considération les intentions qui sont à la base de l'adaptation.

Ces transformations intentionnelles au niveau du sens, et en particulier au niveau de l'idéologie inscrite dans l'œuvre, permettent de poser l'hypothèse que l'adaptation filmique du roman participe à ce qu'on pourrait appeler la « dissimulation idéologique » en fonction de la conjoncture sociale et politique en place. Dissimuler veut dire «cacher» mais aussi «masquer sous des fausses apparences $»^{9}$. Or, l'adaptation, en tant qu'hypertexte dont les sens se construisent par rapport à ceux du texte littéraire comme hypotexte, peut «dissimuler» l'idéologie inscrite dans le roman de différentes manières. D'une part, le film peut atténuer la portée idéologique du roman et ne prendre du texte engagé politiquement ou socialement qu'une histoire à raconter en images, en omettant les événements ou les réflexions qui se réfèrent au contexte sociopolitique ou historique. D'autre part, le film peut utiliser un texte canonique pour se délivrer de son message et exprimer des idées nouvelles, en escamotant l'idéologie qui oriente le roman au sens d'hypotexte et en la remplaçant par une autre, celle du cinéaste ou de son époque ${ }^{10}$. L'analyse des écarts par rapport à l'original littéraire et à son discours idéologique aura pour but de voir quelles sont les transformations apportées et comment elles contribuent à l'atténuation du message du roman ou à une prise de position idéologique différente.

\section{Bonheur d'occasion (1945) de Gabrielle Roy et son adaptation par Claude Fournier (1983) : l'émotionnel et l'identitaire au lieu du politique}

«Roman de passage» (BEAUDET, $2010: 8$ ) entre le roman du terroir et celui de la ville et de la modernité, Bonheur d'occasion (1945) de Gabrielle Roy présente les changements sociaux tels que l'urbanisation et l'américanisation de la société canadienne-française dans les années 1940. Il raconte l'appauvrissement progressif de la famille Lacasse qui vit difficilement le clivage entre les

\footnotetext{
${ }_{9}^{9}$ Voir la définition du verbe «dissimuler » selon Trésor de la langue française : http://atilf.atilf.fr/, date de consultation du site : le 15 juillet 2017.

${ }^{10} \mathrm{La}$ «dissimulation » n'est pas l'unique possibilité de traiter le contenu idéologique du roman par le film qui peut aussi renforcer l'orientation idéologique du texte littéraire. Gina FrEITAG et André LOISELLE (2013 : 194) montrent par exemple comment les adaptations des romans d'horreur non seulement gardent le message idéologique des textes littéraires mais aussi le renforcent par les moyens cinématographiques.
} 
anglophones et les francophones de Montréal, clivage social aussi bien qu'économique. Au déclassement des Lacasse s'oppose leur fille aînée, Florentine, fascinée par la vie urbaine, sa richesse et les possibilités d'autonomie qu'elle offre. Son amour pour Jean Levesque, jeune Canadien français dont le rêve est de monter à tout prix dans l'échelle sociale, n'est pas réciproque. La jeune fille, portant l'enfant de Jean, décide d'épouser l'ami de son amoureux, Emmanuel, qui s'engage et part à la guerre. Le roman de Roy s'inscrit dans le discours idéologique et identitaire des années 1940 et dans ses débats autour de la Seconde Guerre mondiale et de l'enrôlement, présents surtout dans les dialogues entre les personnages. Il présente aussi des clivages sociaux et économiques dans la ville de Montréal où le quartier francophone de Saint-Henri s'oppose par sa pauvreté et son enfermement au quartier anglophone de Westmount qui représente la richesse et l'ouverture au monde et à la modernisation.

Le roman a été porté à l'écran en 1983 par Claude Fournier qui, tout en reprenant l'histoire racontée par Roy ainsi que les personnages principaux du livre, effectue certaines transformations de l'orientation idéologique du texte. De manière générale, le film atténue la portée idéologique du roman et omet la majorité des références au contexte politique et social contemporain à l'écriture du livre. Si le contexte est présent en arrière-plan dans les images (par exemple dans la scène où un groupe de soldats marchent en rangs dans la rue), son rôle est avant tout référentiel. Le réalisateur illustre ainsi le temps historique de l'action. Par contre, les débats sur la guerre et la circonscription sont omis. D'autres changements concernent la représentation de l'espace. Fournier élimine les restaurants populaires - les Deux Records et le bistrot de Madame Philiber espaces qui dans le roman étaient des lieux de rencontre et de discussion sur des sujets sociaux et politiques ${ }^{11}$.

Ces deux espaces absents dans le film ont, dans le roman, une valeur identitaire. Selon Yannick REISCH ces bistrots sont caractéristiques de l'univers du faubourg et du quartier en tant qu' «espace sécurisant et protecteur » $(1978: 254)$. Ils fonctionnent en contraste avec les restaurants modernes et impersonnels tels que le bar-magasin de fast-food «Quinze Cents » où travaille l'héroïne du roman. Les restaurants populaires échappent à la commercialisation, les relations humaines y persistent et les personnages trouvent la liberté de s'exprimer. Au niveau idéologique, ils permettent d'introduire la problématique politique et sociale en tant que lieux de rencontre où les personnages expriment leurs idées.

Les «Deux Records » sert de lieu de réunion pour les patriotes qui discutent de la guerre et de l'enrôlement. Ils y expriment la nécessité de défendre la France - mère-patrie et soulignent l'attachement aux valeurs canadiennes-françaises ainsi que le lien entre le Québec et la France. Le restaurant de Madame Philibert, quant à lui, est un lieu d'accueil pour des marginaux rejetés par la société. La portée critique de cet espace passe d'une part par les conversations entre les personnages

\footnotetext{
${ }^{11}$ Ce changement s'avère important si l'on prend en compte le rôle de l'espace dans le roman de Roy. Selon Hilligje VAN'T LAND (2010 : 96), la structuration spatiale inscrite dans le roman de Roy est un moyen d'exprimer une vision du monde, l'espace étant doté des «valeurs autres » qui dépassent ses fonctions classiques dans le roman réaliste (fonction représentative et référentielle). L'espace est ainsi envisagé comme un système de valeurs idéologiques.
} 
qui expriment leur sentiment de marginalisation, d'autre part par les descriptions où est mis en avant la pauvreté :

Il [Jean] s'approcha de cette devanture embuée et, entre les cartons-affiches, il aperçut au fond, dans un tout petit carré libre, le visage épanoui et rose de la mère Philibert, propriétaire de l'établissement. Elle se tenait derrière le comptoir, juchée sur un haut tabouret, et d'une main caressait un chat noir dont la queue battait le bois poli et usé. Des vêtements mouillés, casquettes et gants jetés sur la tôle qui servait de pare-étincelles, s'échappait une buée grasse et chaude où tremblaient les visages. [...] Au fond de la boutique, il y avait peut-être deux ou trois nouveaux venus dont il apercevait mal le visage ; on amenait parfois un invité aux réunions du soir, des fileurs tout frais embauchés, ou encore quelques jeunes chômeurs que la mère Philibert accueillait avec le même empressement que ses bons clients. Son débit était depuis longtemps le refuge d'une petite troupe bruyante, chicanière et le plus souvent sans le sou. (Roy 1993 : 3233)

Cette description pourrait pourtant bien être transposée dans le « langage » des images. Les omissions évoquées ne semblent donc pas imposées par le changement de média et s'expliquent par l'inscription du film dans le contexte de sa réalisation ${ }^{12}$. Comme les questions de la guerre et de l'engagement militaire, ainsi que celles de l'exclusion sociale des Canadiens français ne sont plus au premier plan en 1983, l'adaptation de Fournier passe sous silence ces sujets ${ }^{13}$. Il reste que l'élimination des questions politiques constitue un changement important par rapport au texte original et influence l'interprétation de Bonheur d'occasion de Fournier.

Ainsi, l'adaptation non seulement change la caractérisation des personnages ${ }^{14}$ mais aussi réduit la portée idéologique du texte, de sorte qu'on peut parler de la «dépolitisation » et de la «mélodramatisation» du roman. Selon Ben-Z. SHEK, l'omission des éléments qu'il qualifie d'essentiels du roman, tels les répercussions collectives de la crise économique de 1929 et le «"salut" ironique » (1984: 482) de la Deuxième Guerre mondiale atténue la critique sociale du roman. Le critique conclut : "C'est pourquoi le résultat global de la lecture de Bonheur d'occasion pratiquée par Claude Fournier contribue à faire du roman un mélodrame [...]» (1984: 483).

La «mélodramatisation » ainsi que la mise en avant de l'amour et du personnage féminin est réalisée dans le film par différents moyens : bande sonore où une chanson revient dans les moments de la tension émotionnelle de l'héroïne, gros plans qui se focalisent sur les visages des personnages. Le

\footnotetext{
${ }^{12}$ Le réalisateur compense au moins partiellement ces omissions en transformant certaines scènes du roman et en en ajoutant des nouvelles, comme celle de la conversation de Rose-Anna avec le propriétaire du truc d'Azarius. La femme se rend chez le propriétaire du camion pour faire regagner l'emploi à son mari. La scène exprime l'opposition entre les Francophones et les Anglophones et souligne la domination économique de ces derniers. Le discours presque monologique de Rose-Anna peut être considéré comme équivalent des conversations dans le restaurant de Madame Philibert.

${ }^{13}$ Christian PoIRIER a remarqué que dans le film de Fournier: « [...] la politique est totalement absente des préoccupations des personnages, malgré le contexte de la Seconde Guerre. Les personnages, à cet égard, semblent subir une Histoire qui se déroule malgré eux. Le film ne fait aucune mention du débat sur la conscription » (2004: 166).

${ }^{14}$ Ben-Z. SHEK constate que Fournier banalise les personnages d'Emmanuel et d'Azarius en leur enlevant toutes les préoccupations de caractère social et politique (1984 : 482).
} 
cinéaste insiste sur ce côté émotionnel du film dans la partie finale de l'adaptation. En fait, le roman finit par la description de l'espace observé par Emmanuel qui partait pour l'Europe, ce qui souligne la portée critique du roman envers la guerre :

Le jeune homme y leva le regard avec une expression de muette pitié. Puis la lumière était loin derrière lui, le train franchissait la place Saint-Henri.

Le visage collé à la vitre, Emmanuel vit fuir les barrières du passage à niveau, le Sacré-Cour de bronze, l'église, la cabine de l'aiguilleur montée sur pilotis. Il aperçut un arbre dans un fond de cour, qui poussait ses branches tordues entre les fils électriques et un réseau de cordes à linge. Ses feuilles dures et ratatinées semblaient à demi mortes de fatigue avant même de s'être pleinement ouvertes.

Très bas dans le ciel, des nuées sombres annonçaient l'orage (Roy, 1993 : 405).

Par contre, la fin du film se focalise sur le personnage de Florentine. Le plan large qui passe au plan américain présente la jeune femme dont le regard s'arrête sur une poussette, ce qui suggère sa maternité future. L'ambiance pessimiste du roman est remplacée par l'espoir dans l'avenir: Florentine est arrivée à s'arracher du cercle familial et est sur le point de commencer une nouvelle vie.

En transformant de cette manière la fin du texte, le film perd un peu de la force contestataire du roman et semble suivre les goûts du public pour les fins heureuses pour s'assurer du succès du film. À part ces raisons économiques, qui sont d'ailleurs d'une grande importance dans le contexte du cinéma, on peut considérer la mise en avant du personnage de Florentine ainsi que la décision de finir le film par les images d'espoir comme des choix intentionnels pour changer l'orientation idéologique du roman.

L'adaptation de Fournier témoigne de la volonté d'accorder au film une portée idéologique différente où sont mis en relief l'aspect identitaire et les questions sociales des années 1980, marquées, entre autres, par l'échec au premier référendum sur la souveraineté du Québec. Le réalisateur renforce la portée identitaire de certains fragments du roman. Par exemple, en mettant à l'écran la scène du départ aux sucres, chez la famille de Rose-Anna, l'auteur insiste sur l'idéalisation de la campagne. Fournier reprend ainsi l'opposition récurrente dans la production culturelle québécoise, entre l'espace urbain et rural. Dans l'interprétation traditionnelle, voire conservatrice, cette opposition n'était pas uniquement celle des lieux mais avait une forte connotation identitaire. Elle confrontait les valeurs telles que industriel-campagnard, protestant-catholique, anglophone-francophone (KWATERKO, 2003 : 79). Dans le roman, le départ a un caractère ambivalent: d'un côté, c'est le retour aux origines, à la famille, de l'autre, un moment de confrontation difficile avec la réalité. La scène permet d'accuser la pauvreté de la population urbaine et la désillusion d'une vie qui n'a pas réalisé les espoirs liés au déménagement dans une ville ${ }^{15}$. Dans le film, l'espace rural est présenté comme un lieu de joie. L'idéalisation est réalisée à l'aide de la bande sonore où la musique vive se substitue aux paroles. Le cinéaste

\footnotetext{
${ }^{15}$ Dans le roman, la joie des personnages se trouve minimisée par la déception de Rose-Anna par l'accueil de la famille et l'attitude critique de sa mère ainsi que ses commentaires sur la pauvreté des enfants élevés en ville.
} 
renoue de cette manière avec l'identité canadienne-française d'avant la Révolution tranquille ${ }^{16}$. Ainsi, tout en atténuant la portée idéologique du texte de départ jusqu'à faire du roman un mélodrame - l'adaptation se charge du discours social et identitaire contemporain à sa réalisation, celui de la redéfinition de l'identité québécoise.

\section{Maria Chapdelaine (1916) de Louis Hémon et son adaptation par Gilles Carle (1983) : s'exprimer en adaptant}

Le film de Fournier, tout en proposant une prise de position dans le contexte contemporain, ne s'oppose pas pour autant au message idéologique de l'original littéraire. Une autre stratégie d'adaptation et de positionnement face à l'idéologie inscrite dans le texte de départ est illustrée par l'adaptation de l'œuvre de Louis Hémon Maria Chapdelaine. Ce roman, publié d'abord en feuilleton dans la revue parisienne Le Temps en 1914 et sous forme de livre à Montréal en 1916, trace l'image de la communauté canadienne-française du début du $\mathrm{XX}^{\mathrm{e}}$ siècle. Maria, fille aînée de la famille Chapdelaine, défricheurs habitant aux alentours du village de Péribonka, est sur le point d'être mariée. La jeune femme est courtisée par trois candidats qui incarnent des styles de vie et valeurs différents : François Paradis, qui vit de divers travaux auxquels il s'emploie dans les forêts, s'inscrit dans la tradition des premiers colonisateurs du Québec, «coureurs de bois » et symbolise la relation avec la nature; Lorenzo Surprenant, revenu à Péribonka pour les obsèques de son père, décide de vendre la terre et de revenir aux États-Unis pour s'y établir - il incarne l'américanisation et la vie urbaine ; Eutrope Gagnant, voisin des Chapdelaine et défricheur comme eux, est attaché à la terre et à la vie rurale. Maria tombe amoureuse de François mais lorsque celui-ci est mort sur la route de la forêt à Péribonka, la jeune femme décide d'épouser Eutrope. Son choix est dû d'une part à la mort de sa mère ; d'autre part, les « voix des ancêtres » l'incitent à rester fidèle aux valeurs du passé.

Déjà le roman lui-même pose différents problèmes pour l'analyse de l'idéologie inscrite dans le texte. Selon Nicole DeschamPS, l'œuvre d'Hémon, écrivain français, devient un texte crucial pour le discours identitaire des Canadiens français, une sorte de roman national, jusqu'à se transformer en mythe :

Écrit et lu au début du vingtième siècle, Maria Chapdelaine rappelle déjà ces contes traditionnels dont on a oublié depuis longtemps la lettre et qui, à force d'être racontés, traduits, interprétés, reécrits, ont acquis dans diverses conjonctures historiques la densité des mythes (1980:8).

\footnotetext{
${ }^{16}$ Une autre transformation qui s'inscrit dans la même stratégie concerne la scène du sermon à l'Église. Les paroles du prêtre remplacent le discours d'Azarius que celui-ci a prononcé dans les «Deux-Records ». Si le réalisateur garde de cette manière le discours sur la nécessité de protéger la France et sur l'attachement aux valeurs canadiennes-françaises (et sur la partie «française » de l'identité québécoise), il redonne de l'importance à l'Église dont le rôle, après une forte contestation pendant la Révolution tranquille, n'est plus le même dans le Québec fortement laïcisé après la Révolution tranquille des années 1960.
} 
En prolongeant cette réflexion, Normand VILLENEUVE (1980: 9) remarque que le roman subit des interprétations qui s'éloignent souvent du texte ou du projet idéologique de l'auteur. Alors que l'œuvre d'Hémon était loin d'idéaliser la réalité des défricheurs, mettant en relief plutôt les aspects non idylliques de la vie dure au sein de la nature hostile, on y voit une image parfaite de la vie traditionnelle des Canadiens français. Le chercheur constate que l'élite canadienne-française, fidèle à l'idéologie de la survivance, s'est approprié le roman pour en faire «un plaidoyer pour le maintien d'une société traditionnelle» (VILLENEUVE, 1980 : 187). Villeneuve situe la fin du mythe de Maria Chapdelaine dans les années 1950, où avec les transformations qui préparent la Révolution Tranquille, on rejette les valeurs que prônait le roman (1980:214). Cela s'accompagne d'un retour au texte qui sera interprété de nouveau.

L'adaptation du roman d'Hémon, réalisée par Gilles Carle en 1983, s’inscrit dans ces réinterprétations. Le réalisateur effectue plusieurs transformations du texte de départ de sorte que son film entre en dialogue avec l'œuvre littéraire et ses lectures précédentes. D'abord, Carle met en relief le personnage de Maria. Sans en faire un personnage focalisateur ou narrateur du film, le réalisateur privilégie l'identification avec la jeune femme. Celle-ci est présente sur l'écran dès le début du film (sous les génériques) et est mentionnée dans les dialogues par les autres personnages. Contrairement au roman qui commence par la description du paysage et la sortie de la messe des personnages masculins, le film s'ouvre par la scène où les parents de Maria discutent de son proche retour. L'ajout d'une scène montrant la traversée du lac permet de suivre le personnage féminin en route vers Péribonka.

La subjectivité de Maria est favorisée également par les monologues intérieurs prononcés en forme de voix off par l'actrice. Cela participe à l'autonomisation du personnage féminin qui dans le roman gardait le plus souvent le silence, ses pensées étant rapportées par le narrateur. L'adaptation rend audibles les paroles de la jeune femme silencieuse du roman et de cette manière lui donne le droit de s'exprimer : Maria de Carle est à la fois plus active et plus autonome que l'héroïne d'Hémon. Elle exprime ses émotions de manière explicite. Le personnage littéraire restait muet à la nouvelle de la mort de François Paradis ${ }^{17}$. Par contre, l'héroïne filmique pleure dans les bras d'Eutrope ${ }^{18}$. Les émotions, qui dans le roman sont imposées à la jeune femme, dans le film lui appartiennent.

Les différences entre le texte de départ et son adaptation sont perceptibles aussi au niveau de l'histoire racontée. Or, Carle modifie la chronologie des événements. Alors que dans le roman la disparition de François Paradis précède la mort de la mère de Maria, dans le film la chronologie est inversée. Ainsi, si la jeune femme refuse le départ aux États-Unis avec Lorenzo, cela ne veut pas dire

\footnotetext{
17 «[...] mais elle ne pouvait parler, ni pour louer le mort, ni pour le plaindre. Une main s'était glissée dans sa gorge, l'étouffant, dès que le dénouement du récit tragique était devenu clair pour elle, et maintenant cette main avait pénétré jusqu'en sa poitrine et lui serrait durement le cœur » (HEMON, 1954 : 141-142).

${ }^{18}$ De même, dans la scène filmique de la messe, elle refuse d'un geste net la proposition de Lorenzo qui voulait l'emmener avec lui. Elle rejette également Eutrope qui, dans le roman, devait interpréter lui-même le silence de la jeune femme.
} 
qu'elle désire remplacer sa mère. Elle garde toujours l'espoir de partir avec François.

C'est la fin du film qui est révélatrice des transformations les plus importantes au niveau de signification. La Maria de Carle finit, elle aussi, par épouser Eutrope et, ainsi, prolonger la tradition. Pourtant, elle le fait de son propre gré. Or, le réalisateur élimine les «voix des ancêtres », cruciales pour les interprétations précédentes du roman :

Alors une troisième voix plus grande que les autres s'éleva dans le silence : la voix du pays de Québec, qui était à moitié un chant de femme et à moitié un sermon de prêtre. [...] Elle disait :

« Nous sommes venus il y a trois cents ans, et nous sommes restés... Ceux qui nous ont menés ici pourraient revenir parmi nous sans amertume et sans chagrin, car s'il est vrai que nous n'ayons guère appris, assurément nous n'avons rien oublié.

Nous avions apporté d'outre-mer nos prières et nos chansons : elles sont toujours les mêmes. Nous avions apporté dans nos poitrines le cœur des hommes de notre pays, vaillant et vif, aussi prompt à la pitié qu'au rire, le cœur le plus humain de tous les cœurs humains : il n'a pas changé. Nous avons marqué un plan du continent nouveau, de Gaspé à Montréal, de Saint-Jeand'Iberville à l'Ungava, en disant : ici toutes les choses que nous avons apportées avec nous, notre culte, notre langue, nos vertus et jusqu'à nos faiblesses deviennent des choses sacrées intangibles et qui devront demeurer jusqu'à la fin.

Autour de nous des étrangers sont venus, qu'il nous plaît d'appeler des barbares ; ils ont pris presque tout le pouvoir; ils ont acquis presque tout l'argent ; mais au pays du Québec rien n'a changé. Rien ne changera, parce que nous sommes un témoignage. De nous-mêmes et de nos destinées nous n'avons compris clairement que ce devoir-là : persister... nous maintenir... Et nous nous sommes maintenus, peut-être afin que dans plusieurs siècles encore le monde se tourne vers nous et dise : "Ces gens sont d'une race qui ne sait pas mourir..." Nous sommes un témoignage.

C'est pourquoi il faut rester dans la province où nos pères sont restés, et vivre comme ils ont vécu, pour obéir au commandement inexprimé qui s'est formé dans leurs cœurs, qui a passé dans les nôtres et que nous devrons transmettre à notre tour à de nombreux enfants : Au pays du Québec rien ne doit mourir et rien ne doit changer... » (HEMON, $1954: 239-241)^{19}$.

Carle clôt le film par la scène où Maria accepte le mariage avec Eutrope, ce qui constitue une adaptation fidèle par rapport au roman. Celui-ci s'achève par la réponse de Maria à Eutrope : «Oui... Si vous voulez, je vous marierai comme vous m'avez demandé, le printemps d'après ce printemps-ci, quand les hommes reviendront du bois pour les semailles »(HEMON, 1954 : 245). Si l'adaptation reprend cette réplique, elle en ajoute une autre et les dernières paroles dans le film sont «Oui, je reste» prononcées par la voix intérieure de la jeune femme. La focalisation de la caméra sur Maria qui semble s'adresser au spectateur suggère l'importance de ces mots et invite à une interprétation.

Les transformations effectuées par Carle font non seulement de Maria Chapdelaine un texte plus «féminin », pour ne pas dire féministe, le personnage principal étant une femme autonome qui prend ses décisions elle-même et ne se laisse pas guider par les «voix » extérieures. Ces modifications sont également révélatrices de l'idéologie de laquelle participe le film et qui diffère de celle du roman. Or, Carle se distancie à la fois de l'identité et de l'attitude des Canadiens français telles que présentées dans le texte littéraire. Il refuse la passivité de la

${ }^{19}$ Carle en cite un fragment dans les génériques au début du film mais omet le fragment sur le caractère fixe du Québec. 
collectivité qui s'enfermait dans les valeurs traditionnelles et dans le passéisme et propose aux Québécois d'adopter une attitude active, de responsabilité, sans rompre totalement avec les valeurs anciennes. On peut voir dans le film de Carle une tentative de se retrouver dans une situation sociale et politique nouvelle, après l'échec du premier référendum sur la souveraineté de la province du Québec. L'auteur propose d'assumer cette situation non pas comme imposée de l'extérieur mais comme résultant de la décision de la société québécoise elle-même.

Ainsi, l'adaptation proposée par Gilles Carle peut être considérée comme une dissimulation, sous un texte ancré dans la tradition et dans l'idéologie conservatrice, des sens qui dépassent les idées et valeurs traditionnelles et en proposent une réinterprétation. Les libertés que prend le réalisateur envers le texte de départ permettent de donner à l'œuvre filmique un sens nouveau, renforcé par son lien avec l'original littéraire. En fait, s'il peut être regardé comme une œuvre autonome, le film de Carle n'acquiert sa pleine signification que par rapport au roman dont il est une relecture.

Si la stratégie de Carle qui dialogue avec le roman diffère de celle de Fournier qui ne fait qu'atténuer certains aspects du message idéologique de l'original littéraire et les remplacer par des contenus plus contemporains, les deux films travaillent l'idéologie du texte littéraire par les mêmes moyens. La dissimulation idéologique s'effectue par des modifications au niveau de la représentation de l'espace et des personnages et au niveau du récit, par les omissions et les ajouts de scènes ou de parties dialogiques. Dans les deux cas, le changement du discours idéologique du roman, s'il participe à l'ensemble des transformations liées à la mise sur l'écran d'un texte littéraire, doit être considéré comme une transformation intentionnelle. Elle vient d'une part de la volonté de suivre les goûts du public et d'omettre ce qui pourrait être incompréhensible aux spectateurs. L'adaptation du texte littéraire au cinéma est ainsi en même temps son adaptation au contexte contemporain, qui rend le texte plus proche et parfois plus «populaire». D' autre part, les cinéastes changent l'idéologie du texte pour s'exprimer et véhiculer leur propre message. Celui-ci est dissimulé dans le film qui se dit être une adaptation du texte littéraire mais qui en réalité l'utilise comme texte de départ et prétexte à une prise de position différente sur les sujets traités dans le roman, voire une expression sur les questions absentes du texte littéraire. L'adaptation devient ainsi non seulement une réinterprétation et une relecture du roman, révélatrice de l'idéologie de l'auteur du film et du discours social de son époque mais aussi une forme de réécriture. Celle-ci unit, selon Lise GAUVIN (2004 : 12), les pratiques de lecture et de création, la reconnaissance d'un modèle et son détournement. Le statut de l'adaptation comme texte dérivé, en mobilisant la connaissance du texte de départ, s'avère particulièrement riche en possibilités d'expression, l'idéologie de l'œuvre nouvelle entrant en dialogue avec celle de l'œuvre première. 


\section{BIBLIOGRAPHIE}

BEAUDET Marie-Andrée (2010), Présentation, in : BEAUDET Marie-Andrée (éd.), Bonheur d'occasion au pluriel. Lectures et approches critiques, Québec, Éditions Nota bene, p. 7-13.

CAMBron Micheline (1989), Une société, un récit. Discours culturel au Québec (1967-1976), Montréal, Éditions de l'HEXAGONE.

Deleuze Gilles (1985), Cinéma II : Image-temps, Paris, Éditions de Minuit.

DELEUZE Gilles, GUATTARI Félix (1984), Kafka : pour une littérature mineure, Paris, Édition de Minuit.

DeschAMPS Nicole (1980), Avant-propos, in: DesChAMPS Nicole ; HerouX Raymonde ; VILLENEUVE Normand (éd.), Le mythe de Maria Chapdelaine, Montréal, Les Presses de l'Université de Montréal, p. 7-15.

Duchet Claude (1971), Pour une socio-critique ou variations sur un incipit, Littérature, 1, p. 5-14.

FreITAG Gina ; LOISELLE André (2013), Tales of Terror in Québec Popular Cinema: The Rise of the French Language Horror Film since 2000, American Review of Canadian Studies 43/2, Routledge, p. 190-203.

GAUDREAUlt André ; MARION Philippe (2008), Un art de l'emprunt. Les sources intermédiales de l'adaptation, in : PLAMONDON Jean-François, Littérature et cinéma au Québec, 1995-2005: actes du Colloque du Centro universitario di studi quebecchesi, Bologna, Pendragon, p. 13-29.

GAUVIN Lise (2004), Écrire/Réécrire le/au féminin : notes sur une pratique, Études françaises, 40/1, p. 11-28.

Helman Alicja (1998), Twórcza zdrada. Filmowe adaptacje literatury, Poznań.

HEMON Louis (1954/1916), Maria Chapdelaine. Récit du Canada français, Paris, Grasset.

HOPFINGER Maryla (1974), Adaptacje filmowe utworów literackich : problemy teorii i interpretacji (Adaptations cinématographiques des cuvres littéraires: questions de théorie de l'interprétation), Wrocław, PAN (Académie Polonaise des sciences).

KWATERKO Józef (2003), Dialogi z Ameryka. O frankofońskiej literaturze w Québecu i na Karaibach, Kraków, UNIVERSITAS.

KYLOUŠEK Petr (2009), Dimension noétique de l'art, in : KYLOUŠEK Petr ; KOLINSKÁ Klára ; PRAJZNEROVÁ Kateřina ; POSPÍŠIL Tomáš ; VOLDŘICHOVÁ BERÁNKOVÁ Eva ; HORÁK Petr (éd.), Us-them-me : the search for identity in Canadian literature and film. Nous-eux-moi : la quête de l'identité dans la littérature et le cinéma canadiens, Brno, Masarykova Univerzita, 2009, p. 2430.

LEVER Yves (1988), Histoire générale du cinéma au Québec, Montréal, Boréal.

POIRIER Christian (2004), Le cinéma québécois. À la recherche d'une identité?, t. I : L'imaginaire filmique, Québec, Presses de l'Université du Québec.

RESCH Yannick (1978), La Ville et son expression romanesque dans Bonheur d'occasion de Gabrielle Roy, Voix et Images, 4/2, p. 244-257.

RoBIN Régine (1993), Pour une socio-poétique de l'imaginaire social, Discours social, 5/1-2, p. 7-32. 
RoY Gabrielle (1993/1945), Bonheur d'occasion, Montréal, Éditions du Boréal.

SERCEAU Michel (1999), L'adaptation cinématographique des textes littéraires: théories et lectures, Liège, Éditions du CEFAL.

SHEK Ben-Z. (1984), Bonheur d'occasion à l'écran : fidélité ou trahison?, Études littéraires, 17/3, p. 481-497.

VAN'T LAND Hilligje (2010), Analyse sociosémiotique des espaces romanesques dans Bonheur d'occasion, in : BEAUDET Marie-Andrée (éd.), Bonheur d'occasion au pluriel. Lectures et approches critiques, Québec, Éditions Nota bene, p. 91-123.

VILLENEUVE Normand (1980), Maria Chapdelaine : catéchisme de la survivance nationale, in: DESCHAMPS Nicole; HEROUX Raymonde; VILLENEUVE Normand (éd.), Le mythe de Maria Chapdelaine, Montréal, Les Presses de l’Université de Montréal, p. 137-218. 